\title{
Updates on the Efficacy of HPV Vaccines and the Emerging RG1-VLP Vaccination against Infections with Genital and Cutaneous Human Papillomaviruses
}

\section{Mohamed L Elsaie*}

Department of Dermatology and Cutaneous Surgery, University of Miami, Florida, USA

A recent study by Centers for Disease Control and Prevention discovered that the commonness of high-hazard strains in high school young ladies dropped significantly after the antibody was presented in 2006, from 7.2 percent in 2006 to 3.6 percent in 2010. Sadly, numerous folks still oppose having their little girls inoculated. A study distributed in March discovered that 44 percent of folks said in 2010 that they didn't mean to immunize their girls, up from 40 percent in 2008. A few folks expect that immunization may advertise wantonness (the new study discovered no indication of that); some see no compelling reason to inoculate young ladies before they come to be sexually animated, in spite of the fact that inoculation heretofore offers the best insurance [1].

Both Gardasil and Cervarix have been resolved to be profoundly viable at forestalling contamination from HPV strains 16 and 18, which represent $70 \%$ of all cervical diseases. (Cervarix is likewise indicating guarantee at security against three extra HPV strains that reason an additional $12 \%$ of cervical diseases, while Gardasil additionally secures against strains 6 and 11, which are connected to genital warts.) The crux, nonetheless, is to arrive at young ladies before they get laid open to HPV, which under optimal circumstances methods immunizing them before they start to engage in sexual relations. A later study discovered that Gardasil is just $44 \%$ successful in ladies as of recently presented to HPV 16 or 18 , while it is $98 \%$ adequate in ladies with no former presentation.

Currently licensed HPV vaccines provide type-restricted protection to HPV16/18 and thus may not protect against ca. 30\% of cervical cancers caused by additional 14 high-risk HPV types. The second virion protein, the $\mathrm{L} 2$ minor capsid protein has been shown to provide low titer antibodies to a broad spectrum of papillomavirus types.

This year; Scientists from Division of Immunology, Allergy and Infectious Diseases (DIAID), Department of Dermatology, Medical University Vienna devised a workable plan to enhance L2's immunogenicity, by adjusting Hpv16 L1 VLP to presentation monotonously a sort regular L2 balance epitope $(\operatorname{Rg} 1)$ on the capsid surface. Inoculation with Hpv16-Rg1 VLP prompted enduring crosskilling antisera against practically all high-danger mucosal HPV and furnished wide range assurance against mucosal HPV in a vaginal test rodent model. This creation raises the plausibility to create a monovalent Hpv16 L1-L2 VLP based chimeric immunization with broader adequacy contrasted with current multivalent immunizations, and likely at lessened cost which might expedite presentation in poorer nations that convey $80 \%$ of the worldwide load of cervical tumors [2-4].

Further more, cutaneous HPV sorts usually creating skin warts in kids and especially in immune suppressed patients are not focused by current immunizations, as are species beta HPV that may assume an extra part in non-melanoma skin disease (squamous cell and basal unit growths), the most widely recognized malignancies in caucasian populaces. We expect to advance prophylactic immunizations against cutaneous HPV sorts moreover dependent upon chimeric L1-L2 VLP. In the event that demonstrated viable in creature models, this may give a sane to vaccinate youngsters with a cutting edge immunization that ensures against skin warts, ano-genital and oro-pharyngeal growths, and might even decrease improvement of non-melanoma skin diseases. Current prophylactic immunizations don't influence predominant HPV tainting or affected neoplasia. Consequently a restorative antibody to instantly influence common contamination is remarkably alluring $[5,6]$. The viral oncoproteins E6 and E7 are held and communicated in advanced sores and hence serve as focuses for a cell resistant reaction [7].

RG1-VLP holds promise as next-generation vaccine with broad efficacy against the vast majority of relevant mucosal and additional cutaneous HPVs, provides a rationale for childhood vaccination, and adds economic advantage of a single antigen formulation compared with multivalent L1-VLP formulations. As post-licensure data confirm excellent safety profiles for HPV L1-VLP vaccines, we infer that RG1VLP vaccination may prove similarly safe, offering the possibility to evaluate vaccine immunogenicity in early-phase human trials.

\section{References}

1. Kondo K, Ochi H, Matsumoto T, Yoshikawa H, Kanda T (2008) Modification of human papillomavirus-like particle vaccine by insertion of the cross-reactive L2-epitopes. J Med Virol 80: 841-846.

2. Lally A, Casabonne D, Imko-Walczuk B, Newton R, Wojnarowska F (2011) Prevalence of benign cu taneous disease among Oxford renal transplant recipients. J Eur Acad Dermatol Venereol 25: 462-470.

3. Longet S, Schiller JT, Bobst M, Jichlinski P, Nardelli-Haefliger D (2011) A murine genital-challenge model is a sensitive measure of protective antibodies against human papillomavirus infection. J Virol 85: 13253-13259.

4. McLaughlin-Drubin ME, Christensen ND, Meyers C (2004) Propagation infection, and neutralization of authentic HPV16 virus. Virology 322: 213-219.

5. Schellenbacher C, Kwak K, Fink D, Shafti-Keramat S, Huber B, et al. (2013) Efficacy of RG1-VLP Vaccination against Infections with Genital and Cutaneous Human Papillomaviruses. J Invest Dermatol.

6. Markowitz LE, Hariri S, Lin C, Dunne EF, Steinau M, et al. (2013) Reduction in Human Papillomavirus (HPV) Prevalence Among Young Women Following HPV Vaccine Introduction in the United States, National Health and Nutrition Examination Surveys, 2003-2010. J Infect Dis 208: 385-393.

7. Elsaie ML (2013) Vaccines from a Dermatology Perspective: HPV Vaccines. $J$ Vaccines Vaccin 4: e116.

*Corresponding author: Mohamed L Elsaie, Department of Dermatology and Cutaneous Surgery, University of Miami, Florida, USA, E-mail: egydoc77@yahoo.com

Received July 29, 2013; Accepted July 30, 2013; Published July 31, 2013

Citation: Mohamed LE (2013) Updates on the Efficacy of HPV Vaccines and the Emerging RG1-VLP Vaccination against Infections with Genital and Cutaneous Human Papillomaviruses. J Vaccines Vaccin 4: e118. doi: 10.4172/2157$7560.1000 \mathrm{e} 118$

Copyright: (c) 2013 Mohamed LE. This is an open-access article distributed under the terms of the Creative Commons Attribution License, which permits unrestricted use, distribution, and reproduction in any medium, provided the original author and source are credited. 